\title{
Nonlinear High Harmonics Generation in REB-Plasma System
}

\author{
Sherif M. Khalii ${ }^{1,2}$, Mizna M. Al-Enazi ${ }^{1}$ \\ ${ }^{1}$ Faculty of Science, Phys. Dept., Princess Nora Bent Abdurrahman University, Riyadh, KSA \\ ${ }^{2}$ Plasma Physics \& Nuclear Fusion Department, N.R.C., Atomic Energy Authority, Cairo, Egypt \\ E-mail: mohamed_khalil@post.com; skhalil@kacst.edu.sa \\ Received October 20, 2010; revised November 21, 2010; accepted November 25, 2010
}

\begin{abstract}
The interaction of a relativistic electron beam (REB) with inhomogeneous, magneto-active, relativistic warm plasma is theoretically investigated. The nonlinear formation of waves at second and triple frequency at the inlet of the beam into the plasma is investigated. Effects of external static or oscillating magnetic field are considered. Nonlinear effects associated with the generation of second and triple harmonics, play an important role in the process of energy transfer from the beam to the plasma as compared with linear stage.
\end{abstract}

Keywords: Nonlinear Generation - Relativistic Electron Beam - Magnetized, Relativistic, Warm Plasmas

\section{Introduction}

The recent and continuous development in the relativistic electron beam (REB) technology has shown the capability of generating powerful electron energy sources, making REB very useful to controlled thermonuclear fusion research in various ways.

Electron beams have many applications in areas like development of new methods in amplification and generation of electromagnetic waves, acceleration of charged particles in plasma, plasma generation, design of microwave tubes waveguides, explanation of natural phenomena that occur in space and solar plasma, material studies, compact torus formation, generation of $x-$ ray and microwave, etc., The recent development of the relativistic electron beam (REB) technology has shown the capability of generating powerful electron energy sources, making the REB very useful for controlled thermonuclear fusion research. It is not surprising to find up till now a long list of studies and research on beam-plasma interaction and applications, which was also reviewed by many authors, e.g. [1-7].

Multiple harmonics generation by laser-plasma interaction has been widely investigated [8-12]. The generation of harmonics through a nonlinear mechanism driven by bunching at the fundamental has sparked interest as a path toward enhancing and extending the usefulness of an x-ray free-electron laser (FEL) facility [13]. Currently, high-order harmonic generation (HHG) is considered as one of the more efficient technique for producing coherent short-wavelength radiation in a broad spectral range [14-18].

An interaction of an electron beam with inhomogeneous plasma is widely investigated in the near past by many authors, e.g., second harmonics generation and plasma heating by REB [19-25]. Wave excitation by REB is also used to minimize energy losses of waves propagating in waveguides filled with magnetized plasma [26-28].

In our investigation, we consider one-dimensional electrostatic oscillations when the directions of propagation, density gradient, and wave electric field coincide with $x$-axis. In our model we take into consideration the following assumptions

1) The hydrodynamic model applies to both the beam and the plasma.

2) Transient thin plasma layer of width $(a)$ is considered so that the plasma density $n_{o p}$ unperturbed by the wave fields, is arbitrary function of $x$ in the region $0 \leq x \leq a$ and equal to zero in the regions $x \leq 0$ and $x \geq a$.

3) The wavelength of the incident beam is large if compared with the width of the plasma transient layer $(a<<\lambda)$, i.e., $\left|k a / \sqrt{\varepsilon_{b}}<<1\right| \varepsilon_{b}$ is the dielectric constant of beam.

4) Plasma electrons are relativistic, warm, collisionless and magnetized.

5) The relativistic electron beam is homogeneous, 
magnetized and have arbitrary temperature compared to that of plasma electrons.

6) Effects of external static or oscillating magnetic field

$$
\vec{H}_{e x t}=\left\{\begin{array}{l}
\vec{e}_{z} H_{0} \\
\vec{e}_{z} H_{0} e^{-i \omega_{m} t}
\end{array}\right.
$$

\section{Basic Equations and Linear Theory}

According to above assumption we can use the following equations:

Equation of motion with relativistic effect,

$$
\left(\frac{\partial}{\partial t}+\vec{V} \cdot \vec{\nabla}\right) m \vec{V}=-e\left(\vec{E}+\frac{1}{c} \vec{V} \times\left(\vec{H}+\vec{H}_{e x t}\right)\right)-\frac{1}{n} \vec{\nabla} P_{s}
$$

where, $s=p, b$ for plasma and beam, respectively, $P_{s}=K T_{s} n_{s}$ is the pressure with gradient $\vec{\nabla} P_{s}=m V_{T s}^{2} \vec{\nabla} n_{s}$, $n_{s}$ is the plasma/beam density, $K$ is Boltzmann constant, $V_{T s}=\sqrt{K T_{s} / m_{s}}$ is the thermal velocity, $m=\frac{m_{0}}{\sqrt{1-\beta^{2}}}$ is the relativistic mass while $m_{o}$ and $m$ are the rest mass and the mass of electrons respectively, $\beta^{2}=\frac{V_{0}^{2}}{C^{2}}$ and $V_{0}$ the initial electron velocity. All other terms have their usual meaning.

Continuity equation

$$
\frac{\partial n_{s}}{\partial t}+\vec{\nabla}\left(n_{s} \vec{V}_{s}\right)=0
$$

Poisson's equation

$$
\frac{\partial E}{\partial x}=-4 \pi e \sum_{s} n_{s}
$$

As plasma is characterized by a collective process, and according to perturbation theory, we can express the density and velocity of the plasma electrons and beam as:

$$
n_{s}=\sum_{s, \beta} n_{s}^{(\beta)}, \quad \vec{V}_{s}=\sum_{s, \beta} \vec{V}_{s}^{(\beta)}, \quad \beta=0,1,2,3, \ldots \ldots
$$

The superscript 0 indicate unperturbed quantities, while 1, 2, 3 indicate perturbation of first, second, third order, and so on. The same could be applied to the electric field as:

$$
\vec{E}=\sum_{\beta} \vec{E}_{\beta}
$$

We also assume that

$$
\begin{gathered}
\left(\left|n_{s}^{(\beta)}\right|,\left|v_{s}^{(\beta)}\right|\right)>>\left(\left|n_{s}^{(\beta+1)}\right|,\left|v_{s}^{(\beta+1)}\right|\right) \\
\frac{\partial v_{b}^{(0)}}{\partial x}=\frac{\partial v_{b}^{(0)}}{\partial t}=\frac{\partial n_{b}^{(0)}}{\partial t}=0 \text { and } v_{b}^{(0)}>>0
\end{gathered}
$$

\subsection{Cold Plasma - Cold Beam}

For cold magnetized non-relativistic plasma and cold non-magnetized relativistic beam, and on the basis of above assumptions, the first perturbed densities and velocities of beam and plasma reads:

$$
\begin{gathered}
v_{b}^{(1)}=\frac{-e}{m \alpha \gamma v_{b}^{(0)}} e^{\frac{i \omega_{1}}{v_{b}^{(0)}} x} \int E_{1} e^{\frac{-i \omega_{1}}{v_{b}^{(0)}}} d x \\
n_{b}^{(1)}=\frac{e n_{b}^{(0)}}{m \alpha \gamma v_{b}^{(0) 2}} e^{\frac{i \omega_{1}}{v_{b}^{(0)}} x} \int e^{\frac{-i \omega_{1}}{v_{b}^{(0)}} x}\left[E_{1}+\frac{i \omega_{1}}{v_{b}^{(0)}} e^{\frac{i \omega_{1}}{v_{b}^{(0)}} x} \int E_{1} e^{\frac{-i \omega_{1}}{v_{b}^{(0)}}} d x^{\prime}\right] d x \\
n_{p}^{(1)}=\frac{-e}{m \omega_{1} \tilde{\omega}_{1}} \frac{\partial x}{\partial x}\left(n_{p}^{(0)} E_{1}\right)
\end{gathered}
$$

where, $\alpha=1+\gamma^{2}\left(\frac{v_{b}^{(0)}}{c}\right)^{2}, \tilde{\omega}_{1}=\omega_{1}-i \omega_{c}, \quad\left(\omega_{c}=\frac{-e H_{o}}{m c}\right)$ is the electron cyclotron frequency, while $\omega_{1}$ is the fundamental frequency).

Using (6) and (8) into (3), and after lengthy but easy calculations we obtain the following differential equation for the fundamental electric field $E_{1}$ :

$$
\left(v_{b}^{(0)} \frac{\partial}{\partial x}-i \omega_{1}\right)^{2} \varepsilon_{1 m} E_{1}+\omega_{1 b}^{2} E_{1}=0
$$

where, $\varepsilon_{1 M}=1-\frac{\omega_{p}^{2}}{\omega_{1} \tilde{\omega}_{1}}$ is the plasma dielectric, $\omega_{1 b}^{2}=\frac{\omega_{b}^{2}}{\alpha \gamma}$, $\omega_{s}$ is the Langmuir frequency $\omega_{s}^{2}=\frac{4 \pi e^{2} n_{0 s}}{m}$. Introducing $E_{1}(x)=\frac{F_{1}(x)}{\varepsilon_{1 M}(x)} e^{\frac{i \omega_{1}}{v_{b}^{(0)}}}$ into (9), we get

$$
\frac{\partial^{2} F_{1}}{\partial x^{2}}+\frac{\chi_{1 M}^{2}}{\varepsilon_{1 M}} F_{1}=0, \quad \chi_{1 M}^{2}=\frac{\omega_{1 b}^{2}}{v_{b}^{(0) 2}}
$$

In the empty regions $x \leq 0, x \geq a,(10)$ have solutions:

$$
\begin{gathered}
F_{1}=c_{1} e^{i \chi_{1 M} x} ; x \leq 0 \\
F_{1}=c_{2} e^{-i \chi_{1 M}(x-a)} ; x \geq a
\end{gathered}
$$

Inside the plasma layer $0 \leq x \leq a$, solution of (10) reads:

$$
F_{1}=c_{3}, \quad 0 \leq x \leq a
$$


where, $c_{1}, c_{2}, c_{3}$ are constants. To obtain (13), we assumed much small plasma layer width compared to wavelengths, i.e., $\left|\frac{\chi_{1 M}}{\sqrt{\varepsilon_{1 M}}} a\right|<<1$.

\subsection{Warm Plasma - Cold Beam}

Let us now introduce warmness to plasma. Accordingly, relations (7) and (8) read:

$$
\begin{gathered}
v_{p}^{(1)}=\frac{-i e}{m \tilde{\omega}_{1}^{2}} E_{1}-\frac{i v_{t}^{2}}{\tilde{\omega}_{1} n_{p}^{(0)}} \frac{\partial n_{p}^{(0)}}{\partial x} \\
n_{p}^{(1)}=\frac{-e}{m \omega_{1} \tilde{\omega}_{1}} \frac{\partial}{\partial x}\left(n_{p}^{(0)} E_{1}\right) \cdot-\frac{V_{T}^{2}}{\omega_{1} \tilde{\omega}_{1}} \frac{\partial^{2} n_{p}^{(0)}}{\partial x^{2}}
\end{gathered}
$$

Then, (10) converts to:

$$
\frac{\partial^{2} F_{1}}{\partial x^{2}}+\frac{\chi_{1 M T}^{2}}{\varepsilon_{1 M T}} F_{1}=R_{1 T}
$$

where, $\quad \chi_{1 M T}^{2}=\chi_{1 M}^{2}=\frac{\omega_{1 b}^{2}}{v_{b}^{(0) 2}}, \quad \varepsilon_{1 M T}=\varepsilon_{1 M}=1-\frac{\omega_{p}^{2}}{\omega_{1} \tilde{\omega}_{1}}$, $V_{T}=\sqrt{\frac{T_{e}}{m}}$ (thermal velocity), and

$$
R_{1 T}(x)=\frac{4 \pi e}{v_{b}^{(0) 2}} e^{\frac{-i \omega_{1}}{v_{b}^{(0)}}} \frac{V_{T}^{2}}{\omega_{1} \tilde{\omega}_{1}} \frac{\partial}{\partial x}\left(v_{b}^{(0)} \frac{\partial}{\partial x}-i \omega_{1}\right)^{2} n_{p}^{(0)}
$$

It is clear that (16) differs from (10) because of the existence of the source term $R_{1 T}$ due to plasma warmness, which causes an inhomogeneity in the differential equation.

Solutions of (16) in the empty regions, are still given by (10) and (11), while in the inhomogeneous plasma layer, and under the condition $\left|\frac{\chi_{1 M T}}{\sqrt{\varepsilon_{1 M T}}} a\right|<<1$, the solu-

$$
\begin{array}{r}
n_{p}^{(2)}=\frac{\partial}{\partial x}\left\{\frac{-e n_{p}^{(0)}}{m \omega_{2} \tilde{\omega}_{2}} E_{2}+\frac{e^{2} n_{p}^{(0)}\left(\omega_{1}+\tilde{\omega}_{2}\right)}{m^{2} \omega_{1} \tilde{\omega}_{1}^{2} \omega_{2} \tilde{\omega}_{2}} E_{1} \frac{\partial E_{1}}{\partial x}+\left(\frac{e^{2} E_{1}^{2}}{m^{2} \omega_{1} \tilde{\omega}_{1}^{2} \omega_{2}}-\frac{V_{T}^{2}}{\omega_{2} \tilde{\omega}_{2}}\right) \frac{\partial n_{p}^{(0)}}{\partial x}\right\} \\
v_{b}^{(2)}=\frac{-1}{v_{b}^{(0)}} e^{\frac{i \omega_{2}}{v_{b}^{(0)}} x} \int e^{\frac{-i \omega_{2}}{v_{b}^{(0)}} x}\left[\frac{e}{m \gamma} E_{2}+\alpha_{2} v_{b}^{(1)} \frac{\partial v_{b}^{(1)}}{\partial x}+\frac{V_{T}^{2}}{n_{b}^{(0)} \gamma} \frac{\partial n_{b}^{(1)}}{\partial x}\right] d x \\
n_{b}^{(2)}=\frac{-1}{v_{b}^{(0)}} e^{\frac{i \omega_{2}}{v_{b}^{(0)}} x} \int e^{\frac{-i \omega_{2}}{v_{b}^{(0)}} x}\left\{\frac{-n_{b}^{(0)}}{v_{b}^{(0)}} \frac{i \omega_{2}}{v_{b}^{(0)}} e^{\frac{i \omega_{2}}{v_{b}^{(0)}} x} \int e^{\frac{-i \omega_{2}}{v_{b}^{(0)}}} E_{2} d x-\frac{n_{b}^{(0)}}{v_{b}^{(0)}} E_{2}+\frac{\partial}{\partial x}\left(n_{b}^{(1)} v_{b}^{(1)}\right)\right\}
\end{array}
$$

In presence of static magnetic field, and for warm beam warm plasma interaction, the second perturbed quantities (with $\left(\omega_{2}=2 \omega_{1}\right)$ for the plasma and beam reads:

$$
v_{p}^{(2)}=\frac{-i e}{m \tilde{\omega}_{2}} E_{2}+\frac{i e^{2}}{m^{2} \tilde{\omega}_{1}^{2} \tilde{\omega}_{2}} E_{1} \frac{\partial E_{1}}{\partial x}-\frac{i V_{T}^{2}}{n_{p}^{(0)} \tilde{\omega}_{2}} \frac{\partial n_{p}^{(0)}}{\partial x}
$$

Using (19) and (20) into Poisson's Equation (3), we obtain the following differential equation for the second harmonic electric field $E_{2}$ :

$$
\frac{\partial^{2} F_{2}}{\partial x^{2}}+\frac{\chi_{2}^{2}}{\varepsilon_{2 M}} F_{2}=R_{2 M T}^{\prime}
$$


where, $R_{2 M T}^{\prime}$ is a nonlinear source term includes the effects of warmness of both the beam and plasma elec- trons, assuming that both have the same thermal velocity $V_{T}$.

$$
\begin{gathered}
R_{2 M T}^{\prime}(x)=\frac{1}{v_{b}^{(0) 2}} e^{\frac{-i \omega_{2}}{v_{b}^{(0)}}}\left[R_{p M T}+R_{b T}\right], \quad E_{2}=\frac{F_{2}}{\varepsilon_{2 M}} e^{\frac{i \omega_{2}}{v_{b}^{(0)}}}, \quad \chi_{2}^{2}=\frac{\omega_{b}^{2}}{\gamma \alpha_{2} v_{b}^{(0) 2}}, \\
R_{b T}(x)=-4 \pi e\left[\left(i \omega_{2}-v_{b}^{(0)} \frac{\partial}{\partial x}\right) n_{b}^{(1)} v_{b}^{(1)}+n_{b}^{(0)}\left(\alpha_{2} v_{b}^{(1)} \frac{\partial v_{b}^{(1)}}{\partial x}+\frac{V_{T}^{2}}{n_{b}^{(0)} \gamma} \frac{\partial n_{b}^{(1)}}{\partial x}\right)\right] \\
R_{p M T}(x)=-\left(v_{b}^{(0)} \frac{\partial}{\partial x}-i \omega_{2}\right)^{2}\left\{\frac{e \omega_{p}^{2}\left(\omega_{1}+\tilde{\omega}_{2}\right)}{m \omega_{2} \tilde{\omega}_{2} \omega_{1} \tilde{\omega}_{1}^{2}} E_{1} \frac{\partial E_{1}}{\partial x}+\left(\frac{e E_{1}^{2}}{m \tilde{\omega}_{1}^{2} \omega_{1} \omega_{2}}-\frac{m V_{T}^{2}}{e \omega_{1} \tilde{\omega}_{2}}\right) \frac{\partial \omega_{p}^{2}}{\partial x}\right\} \\
\tilde{\omega}_{2}=\omega_{2}-i \omega_{c}, \quad \varepsilon_{2 M}=1-\frac{\omega_{p}^{2}}{\omega_{2} \tilde{\omega}_{2}}, \quad \alpha_{2}=1+2 \gamma^{2}\left(\frac{v_{b}^{(0)}}{c}\right)^{2}
\end{gathered}
$$

Solutions of (22) in the empty regions and in the inhomogeneous plasma layer, are given by:

$$
F_{2}=\frac{e^{i \chi_{2} x}}{2 i \chi_{2}} \int R_{b T} e^{-i \chi_{2} x} d x-\frac{e^{-i \chi_{2} x}}{2 i \chi_{2}} \int_{0}^{x} R_{p M T} e^{i \chi_{2} x} d x, x \leq 0
$$

$$
F_{2}=\frac{e^{i \chi_{2}(x-a)}}{2 i \chi_{2}} \int R_{b T} e^{-i \chi_{2}(x-a)} d x-\frac{e^{-i \chi_{2}(x-a)}}{2 i \chi_{2}} \int_{0}^{x} R_{p M T} e^{i \chi_{2}(x-a)} d x, \quad x \geq a
$$

$$
F_{2} \cong v_{b}^{(0) 2} \int_{0}^{x} d x^{\prime} \int_{0}^{x^{\prime}} R_{2 M T}^{\prime}(x) e^{\frac{-i \omega_{2}}{v_{b}^{(0)}}} d x^{\prime \prime}+c, \quad 0 \leq x \leq a
$$

It is clear that source terms, i.e., $R_{b T}, R_{p M T}$ are represented by products of fundamentals, and the electric field $E_{2}$, as per solutions (23-25), are proportional to waves of second harmonics. This leads to a nonlinear amplification of waves in the inhomogeneous plasma layer and in turn additional plasma heating.

\subsubsection{Second Harmonic Wave Generation in an}

Oscillating Magnetic Field $\vec{H}_{e x t}=\vec{e}_{z} H_{0} e^{-i \omega_{M} \cdot t}$

oscillates with same frequency of fundamental wave, i.e., $\omega_{M}=\omega_{1}$. This oscillating field has no effect in the linear stage. On the other hand it is strongly affect the second order perturbations of warm plasma velocity and density as:

$$
\begin{aligned}
& v_{p}^{(2)}= \\
& \frac{-i e}{m \omega_{2}} E_{2}+\frac{i e^{2}}{m^{2} \omega_{1}^{2} \omega_{2}} E_{1} \frac{\partial E_{1}}{\partial x}-\frac{e \omega_{c}(t)}{m \omega_{1} \omega_{2}} E_{1}-\frac{i V_{T}^{2}}{n_{p}^{(0)} \omega_{2}} \frac{\partial n_{p}^{(0)}}{\partial x}
\end{aligned}
$$

Let us consider here the effect of external magnetic field

$$
n_{p}^{(2)}=\frac{\partial}{\partial x}\left\{\frac{-e n_{p}^{(0)}}{m \omega_{2}^{2}} E_{2}+\frac{e^{2} n_{p}^{(0)}\left(\omega_{1}+\omega_{2}\right)}{m^{2} \omega_{1}^{3} \omega_{2}^{2}} E_{1} \frac{\partial E_{1}}{\partial x}+\left(\frac{e^{2} E_{1}^{2}}{m^{2} \omega_{1}^{3} \omega_{2}}-\frac{V_{T}^{2}}{\omega_{2}^{2}}\right) \frac{\partial n_{p}^{(0)}}{\partial x}+\frac{i e n_{p}^{(0)} \omega_{c}(t)}{m c \omega_{1} \omega_{2}^{2}} E_{1}\right\}
$$

When we look for harmonic generation with $\omega_{2}=2 \omega_{1}$, the perturbed cold electron beam perturbations reads:

$$
v_{b}^{(2)}=\frac{-1}{v_{b}^{(0)}} e^{\frac{i \omega_{2}}{v_{b}^{(0)} x}} \int e^{\frac{-i \omega_{2}}{v_{b}^{(0)}}}\left[\frac{e}{m \gamma} E_{2}+\alpha_{2} v_{b}^{(1)} \frac{\partial v_{b}^{(1)}}{\partial x}\right] d x
$$

$$
\begin{aligned}
n_{b}^{(2)}= & \frac{-1}{v_{b}^{(0)}} e^{\frac{i \omega_{2}}{v_{b}^{(0)}} x} \int e^{\frac{-i \omega_{2}}{v_{b}^{(0)}} x}\left\{\frac{-n_{b}^{(0)}}{v_{b}^{(0)}} \frac{i \omega_{2}}{v_{b}^{(0)}} e^{\frac{i \omega_{2}}{v_{b}^{(0)}} x} \int e^{\frac{-i \omega_{2}}{v_{b}^{(0)}}}\left[\frac{e}{m \gamma} E_{2}+\alpha v_{b}^{(1)} \frac{\partial v_{b}^{(1)}}{\partial x}\right] d x\right. \\
& \left.-\frac{n_{b}^{(0)}}{v_{b}^{(0)}}\left[\frac{e}{m \gamma} E_{2}+\alpha_{2} v_{b}^{(1)} \frac{\partial v_{b}^{(1)}}{\partial x}\right]+\frac{\partial}{\partial x}\left(n_{b}^{(1)} v_{b}^{(1)}\right)\right\} d x
\end{aligned}
$$


Accordingly, the differential equation governing the electric field is given by:

$$
\begin{aligned}
& y \text {, the differential equation governing the } \\
& \text { is given by: } \\
& \frac{\partial^{2} F_{2}}{\partial x^{2}}+\frac{\chi_{2}^{2}}{\varepsilon_{2}} F_{2}=R_{2 M O T} \quad \text { where, } R_{2 M O T}=\frac{1}{v_{b}^{(0) 2}} e^{\frac{-i \omega_{2}}{v_{b}^{(0)}}}\left[R_{p M O T}+R_{b}\right] \\
& R_{p M O T}=-\left(v_{b}^{(0)} \frac{\partial}{\partial x}-i \omega_{2}\right)^{2}\left[\frac{e \omega_{p}^{2}\left(\omega_{1}+\omega_{2}\right)}{m \omega_{1}^{3} \omega_{2}^{2}} E_{1} \frac{\partial E_{1}}{\partial x}+\left(\frac{e E_{1}^{2}}{m \omega_{1}^{3} \omega_{2}}-\frac{m V_{T}^{2}}{e \omega_{1} \omega_{2}}\right) \frac{\partial \omega_{p}^{2}}{\partial x}+\frac{i \omega_{p}^{2} \omega_{c}(t)}{\omega_{1} \omega_{2}^{2}} E_{1}^{2}\right]
\end{aligned}
$$

$$
\begin{array}{r}
F_{2}=\frac{e^{i \chi_{2} x}}{2 i \chi_{2}} \int R_{b} e^{-i \chi_{2} x} d x-\frac{e^{-i \chi_{2} x}}{2 i \chi_{2}} \int_{0}^{x} R_{p M O T} e^{i \chi_{2} x} d x, x \leq 0 \\
F_{2}=\frac{e^{i \chi_{2}(x-a)}}{2 i \chi_{2}} \int R_{b} e^{-i \chi_{2}(x-a)} d x-\frac{e^{-i \chi_{2}(x-a)}}{2 i \chi_{2}} \int_{0}^{x} R_{p M O T} e^{i \chi_{2}(x-a)} d x, \quad x \geq a
\end{array}
$$

$F_{2} \cong \int_{0}^{x} d x^{\prime} \int_{0}^{x^{\prime}}\left(R_{b}+R_{p M O T}\right) e^{\frac{-i \omega_{2}}{v_{b}^{(0)}}} d x^{\prime \prime}+c, 0 \leq x \leq a$

\subsubsection{Third Harmonic Wave Generation in Static \\ Magnetic Field $\vec{H}_{e x t}=\vec{e}_{z} H_{0}$}

In absence of magnetic field, and for cold beam - cold plasma interaction, the third perturbed quantities with $\left(\omega_{3}=3 \omega_{1}\right)$ for the plasma and beam reads:

$$
v_{b}^{(3)}=\frac{-1}{v_{b}^{(0)}} e^{\frac{i \omega_{3}}{v_{b}^{(0)} x}} \int e^{\frac{-i \omega_{3}}{v_{b}^{(0)}}}\left[\frac{e}{m \gamma} E_{3}+\alpha v_{b}^{(2)} \frac{\partial v_{b}^{(1)}}{\partial x}+v_{b}^{(1)} \frac{\partial v_{b}^{(2)}}{\partial x}\right] d x
$$

$$
v_{p}^{(3)}=\frac{-i e}{m \omega_{3}} E_{3}-\frac{e E_{1}}{m \omega_{1} \omega_{3}} \frac{\partial}{\partial x}\left[\frac{-i e}{m \omega_{2}} E_{2}+\frac{i e^{2}}{m^{2} \omega_{1}^{2} \omega_{2}} E_{1} \frac{\partial E_{1}}{\partial x}\right]-\frac{e}{m \omega_{1} \omega_{3}}\left[\frac{-i e}{m \omega_{2}} E_{2}+\frac{i e^{2}}{m^{2} \omega_{1}^{2} \omega_{2}} E_{1} \frac{\partial E_{1}}{\partial x}\right] \frac{\partial E_{1}}{\partial x}
$$

$$
\begin{aligned}
n_{p}^{(3)}= & \frac{\partial}{\partial x}\left\{\frac{-e n_{p}^{(0)}}{m \omega_{3}^{2}} E_{3}+\frac{i e n_{p}^{(0)} E_{1}}{m \omega_{1} \omega_{3}^{2}} \frac{\partial}{\partial x} L+\frac{i e n_{p}^{(0)}\left(\omega_{1}+\omega_{3}\right)}{m \omega_{1}^{2} \omega_{3}^{2}} L \frac{\partial E_{1}}{\partial x}+\frac{i e E_{1}}{m \omega_{1}^{2} \omega_{3}} L \frac{\partial n_{p}^{(0)}}{\partial x}\right. \\
& \left.-\frac{e}{m \omega_{1} \omega_{3}} \frac{\partial}{\partial x}\left[\frac{-e n_{p}^{(0)}}{m \omega_{2}^{2}} E_{2}+\frac{e^{2} n_{p}^{(0)}\left(\omega_{1}+\omega_{2}\right)}{m^{2} \omega_{1}^{3} \omega_{2}^{2}} E_{1} \frac{\partial E_{1}}{\partial x}+\frac{e^{2} E_{1}^{2}}{m^{2} \omega_{1}^{3} \omega_{2}} \frac{\partial n_{p}^{(0)}}{\partial x}\right]\right\}
\end{aligned}
$$

Using (35) and (39) into Poisson's Equation (3), the differential equation governing the third harmonic elecwhere,

$$
\frac{\partial^{2} F_{3}}{\partial x^{2}}+\frac{\chi_{3}^{2}}{\varepsilon_{3}} F_{3}=R_{3}(x)
$$

$$
\varepsilon_{3}=1-\frac{\omega_{p}^{2}}{\omega_{3}^{2}}, \quad \chi_{3}^{2}=\frac{\omega_{b}^{2}}{\gamma v_{b}^{(0) 2}}, \quad E_{3}=\frac{F_{3}}{\varepsilon_{3}} e^{\frac{i \omega_{3}}{v_{b}^{(0)} x}}, \quad R_{3}(x)=\frac{1}{v_{b}^{(0) 2}} e^{\frac{-i \omega_{3}}{v_{b}^{(0)}} x}\left[R_{3 p}+R_{3 b}\right]
$$




$$
\begin{gathered}
R_{3 p}(x)=-\left(v_{b}^{(0)} \frac{\partial}{\partial x}-i \omega_{3}\right)^{2}\left\{[ \frac { i \omega _ { p } ^ { 2 } E _ { 1 } } { \omega _ { 1 } \omega _ { 3 } ^ { 2 } } \frac { \partial } { \partial x } + \frac { i \omega _ { p } ^ { 2 } ( \omega _ { 1 } + \omega _ { 3 } ) } { \omega _ { 1 } ^ { 2 } \omega _ { 3 } ^ { 2 } } \frac { \partial E _ { 1 } } { \partial x } + \frac { i E _ { 1 } } { \omega _ { 1 } ^ { 2 } \omega _ { 3 } } \frac { \partial \omega _ { p } ^ { 2 } } { \partial x } ] \left[\frac{-i e}{m \omega_{2}} E_{2}\right.\right. \\
\left.\left.+\frac{i e^{2} E_{1}}{m^{2} \omega_{1}^{2} \omega_{2}} \frac{\partial E_{1}}{\partial x}\right]+\frac{E_{1}}{\omega_{1} \omega_{2}^{2} \omega_{3}} \frac{\partial}{\partial x}\left(\omega_{p}^{2} E_{2}\right)-\frac{E_{1}}{\omega_{1} \omega_{3}} \frac{\partial}{\partial x}\left[\frac{e \omega_{p}^{2}\left(\omega_{1}+\omega_{2}\right)}{m \omega_{1}^{3} \omega_{2}^{2}} E_{1} \frac{\partial E_{1}}{\partial x}+\frac{e E_{1}^{2}}{m \omega_{1}^{3} \omega_{2}} \frac{\partial \omega_{p}^{2}}{\partial x}\right]\right\} \\
R_{3 b}(x)=-4 \pi e\left[\left(i \omega_{3}-v_{b}^{(0)} \frac{\partial}{\partial x}\right)\left(n_{b}^{(1)} v_{b}^{(2)}+n_{b}^{(2)} v_{b}^{(1)}\right)+n_{b}^{(0)}\left(\alpha_{2} v_{b}^{(2)} \frac{\partial v_{b}^{(1)}}{\partial x}+v_{b}^{(1)} \frac{\partial v_{b}^{(2)}}{\partial x}\right)\right]
\end{gathered}
$$

with solutions

$$
\begin{gathered}
F_{3}=\frac{e^{i \chi_{3} x}}{3 i \chi_{3}} \int R_{3 b} e^{-i \chi_{3} x} d x-\frac{e^{-i \chi_{3} x}}{3 i \chi_{3}} \int_{0}^{x} R_{3 p} e^{i \chi_{3} x} d x, x \leq 0 \\
F_{3}=\frac{e^{i \chi_{3}(x-a)}}{3 i \chi_{3}} \int R_{3 b} e^{-i \chi_{3}(x-a)} d x-\frac{e^{-i \chi_{3}(x-a)}}{3 i \chi_{3}} \int_{0}^{x} R_{3 p} e^{i \chi_{3}(x-a)} d x, \quad x \geq a
\end{gathered}
$$

$$
\begin{gathered}
F_{3} \cong \int_{0}^{x} d x^{\prime} \int_{0}^{x^{\prime}}\left(R_{3 b}+R_{3 p}\right) e^{\frac{-i \omega_{3}}{v_{b}^{(0)}} d x^{\prime \prime}+c, 0 \leq x \leq a} \quad \begin{array}{c}
\text { In a static magnetic field, and warm plasma, beam per- } \\
\text { turbations (34) and (35) are still the same, while for } \\
\text { plasma, (36) and (37) reads: }
\end{array} \\
v_{p}^{(3)}=\frac{-i e}{m \tilde{\omega}_{3}} E_{3}-\frac{i V_{T}^{2}}{n_{p}^{(0)} \tilde{\omega}_{3}} \frac{\partial n_{p}^{(0)}}{\partial x}-\frac{e E_{1}}{m \tilde{\omega}_{1} \tilde{\omega}_{3}} \frac{\partial}{\partial x}\left[\frac{-i e}{m \tilde{\omega}_{2}} E_{2}+\frac{i e^{2}}{m^{2} \tilde{\omega}_{1}^{2} \tilde{\omega}_{2}} E_{1} \frac{\partial E_{1}}{\partial x}\right]-\frac{e}{m \tilde{\omega}_{1} \tilde{\omega}_{3}}\left[\frac{-i e}{m \tilde{\omega}_{2}} E_{2}+\frac{i e^{2}}{m^{2} \tilde{\omega}_{1}^{2} \tilde{\omega}_{2}} E_{1} \frac{\partial E_{1}}{\partial x}\right] \frac{\partial E_{1}}{\partial x} \quad(42) \\
n_{p}^{(3)}=\frac{\partial}{\partial x}\left\{\frac{-e n_{p}^{(0)}}{m \tilde{\omega}_{3} \omega_{3}} E_{3}-\frac{V_{T}^{2}}{\tilde{\omega}_{3} \omega_{3}} \frac{\partial n_{p}^{(0)}}{\partial x}+\frac{i e n_{p}^{(0)} E_{1}}{m \tilde{\omega}_{1} \tilde{\omega}_{3} \omega_{3}} \frac{\partial}{\partial x} L_{M}+\frac{i e n_{p}^{(0)}\left(\omega_{1}+\tilde{\omega}_{3}\right)}{m \tilde{\omega}_{1} \omega_{1} \omega_{3}^{2}} L_{M} \frac{\partial E_{1}}{\partial x}+\frac{i e E_{1}}{m \tilde{\omega}_{1} \omega_{1} \omega_{3}} L_{M} \frac{\partial n_{p}^{(0)}}{\partial x}\right. \\
\left.-\frac{e}{m \tilde{\omega}_{1} \omega_{3}} \frac{\partial}{\partial x}\left[\frac{-e n_{p}^{(0)}}{m \tilde{\omega}_{2} \omega_{2}} E_{2}+\frac{e^{2} n_{p}^{(0)}\left(\omega_{1}+\tilde{\omega}_{2}\right)}{m^{2} \tilde{\omega}_{1}^{2} \omega_{1} \tilde{\omega}_{2} \omega_{2}} E_{1} \frac{\partial E_{1}}{\partial x}+\frac{e^{2} E_{1}^{2}}{m^{2} \tilde{\omega}_{1}^{2} \omega_{1} \omega_{2}} \frac{\partial n_{p}^{(0)}}{\partial x}\right]\right\}
\end{gathered}
$$

where, $L_{M}=\frac{-i e}{m \tilde{\omega}_{2}} E_{2}+\frac{i e^{2}}{m^{2} \tilde{\omega}_{1}^{2} \tilde{\omega}_{2}} E_{1} \frac{\partial E_{1}}{\partial x}$

In this case the differential equation governing the third harmonic electric field $E_{3}$ reads

$$
\begin{gathered}
R_{3 M T}(x)=\frac{1}{v_{b}^{(0) 2}} e^{\frac{-i \omega_{3}}{v_{b}^{(0)}}}\left[R_{3 p M T}+R_{3 b}\right], \\
\text { and } R_{3 p M T}(x)=-\left(v_{b}^{(0)} \frac{\partial}{\partial x}-i \omega_{3}\right)^{2}\left\{[ \frac { i \omega _ { p } ^ { 2 } E _ { 1 } } { \tilde { \omega } _ { 1 } \omega _ { 3 } \tilde { \omega } _ { 3 } } \frac { \partial } { \partial x } + \frac { i \omega _ { p } ^ { 2 } ( \omega _ { 1 } + \tilde { \omega } _ { 3 } ) } { \omega _ { 1 } \tilde { \omega } _ { 1 } \omega _ { 3 } } \frac { \partial E _ { 1 } } { \partial x } + \frac { i E _ { 1 } } { \omega _ { 1 } \tilde { \omega } _ { 1 } \omega _ { 3 } } \frac { \partial \omega _ { p } ^ { 2 } } { \partial x } ] \left[\frac{-i e}{m \tilde{\omega}_{2}} E_{2}\right.\right. \\
\left.\left.+\frac{i e^{2} E_{1}}{m^{2} \tilde{\omega}_{1}^{2} \tilde{\omega}_{2}} \frac{\partial E_{1}}{\partial x}\right]-\frac{E_{1}}{\tilde{\omega}_{1} \omega_{3}} \frac{\partial}{\partial x}\left[\frac{-\omega_{p}^{2}}{\omega_{2} \tilde{\omega}_{2}} E_{2}+\frac{e \omega_{p}^{2}\left(\omega_{1}+\tilde{\omega}_{2}\right)}{m \omega_{1} \tilde{\omega}_{1}^{2} \omega_{2} \tilde{\omega}_{2}} E_{1} \frac{\partial E_{1}}{\partial x}+\frac{e E_{1}^{2}}{m \omega_{1} \tilde{\omega}_{1}^{2} \omega_{2}} \frac{\partial \omega_{p}^{2}}{\partial x}\right]-\frac{m V_{T}^{2}}{e \omega_{3} \tilde{\omega}_{3}} \frac{\partial \omega_{p}^{2}}{\partial x}\right\}
\end{gathered}
$$

Solutions of (45) are:

$$
\begin{gathered}
F_{3}=\frac{e^{i \chi_{3} x}}{3 i \chi_{3}} \int R_{3 b} e^{-i \chi_{3} x} d x-\frac{e^{-i \chi_{3} x}}{3 i \chi_{3} x} \int_{0}^{x} R_{3 p M T} e^{i \chi_{3} x} d x, x \leq 0 \\
F_{3}=\frac{e^{i \chi_{3}(x-a)}}{3 i \chi_{3}} \int R_{3 b} e^{-i \chi_{3}(x-a)} d x-\frac{e^{-i \chi_{3}(x-a)}}{3 i \chi_{3}} \int_{0}^{x} R_{3 p M T} e^{i \chi_{3}(x-a)} d x, \quad x \geq a
\end{gathered}
$$




$$
F_{3} \cong \int_{0}^{x} d x^{\prime} \int_{0}^{x^{\prime}}\left(R_{3 b}+R_{3 p M T}\right) e^{\frac{-i \omega_{3}}{v_{b}^{(0)}} x} d x^{\prime \prime}+c, 0 \leq x \leq a
$$

\section{Conclusions}

The nonlinear interaction of a relativistic electron beam (REB) with an inhomogeneous, magneto-active, relativistic warm plasma layer is investigated. The nonlinear effects associated with the generation of second and third harmonics, plays an important role in the process of energy transfer from the beam to the plasma as compared with linear stage. This is due to the fact that the electric field intensity at double and third harmonics is stronger than that of the basic frequency.

Fields are found to be inversely proportional to $\varepsilon$ as:

$$
E_{i} \propto \frac{1}{\varepsilon_{i M}},
$$

where $\varepsilon_{i M}=1-\frac{\omega_{p}^{2}}{\omega_{i} \tilde{\omega}_{i}}, i=1,2,3.1,2$ and 3 indicates fundamental, second and third harmonics respectively and $\tilde{\omega}_{i}=\omega_{i}-i \omega_{c}$.

In static strong magnetic field, at resonance $\left|\tilde{\omega}_{i}\right| \rightarrow 0$, the wave's amplitudes and the electric fields for fundamentals and higher harmonics are sharply increases.

As seen, the amplitudes of the exponentially growing oscillations at frequencies $2 \omega$ and $3 \omega$ are expressed through the amplitudes of the beam Langmuir oscillations in the region where is no plasma present. Thus, once there are Langmuir oscillations in the beam, even in the presence of external magnetic field, their frequency being equal to $\omega$ in the laboratory frame, and the oscillations with frequencies $2 \omega$ and $3 \omega$ are always generated at the inlet of the beam into the plasma when the beam in the plasma is unstable in relation to the excitation of oscillations with frequencies $2 \omega$ and $3 \omega$. This lead to the conclusion that far enough from the plasma boundary inwards, the external magnetic field may increase the amplitude of grounded waves, but still the electric field of waves with double frequency would be stronger than that of basic frequency.

Also we can say that, the nonlinear source terms ( $R_{2 M T}^{\prime} R_{2 M O T}, R_{3}, R_{3 M T}$ ) which is due to nonlinearity, static or oscillating magnetic field effects, and warmness of plasma and beam electrons, are in our case play a crucial role via wave amplification at second and third harmonic.

It is also shown that the nonlinear effects associated with the generation of second and third harmonics, play an important role in the process of energy transfer from the beam to the plasma as compared with linear stage.b This due to the fact that the electric field intensity at higher harmonics is stronger than that of basic frequency.

\section{References}

[1] G. P. Gupta, et al.,"Relativistic Electron Beam Heating of a Hydrogen Plasma in Open Confinement Systems: Theoretical Model," Physics of Fluids, Vol. 31, 1988, p. 606.

[2] G. C. A. M. Janssen, et al., "Plasma Heating by a Relativistic Electron Beam," Physics of Fluids, Vol. 27, 1984, p. 726.

[3] L. E. Thodem, "Effect of Electron-Ion Collisions on the Nonlinear State of the Relativistic Two-Stream Instability," Physics of Fluids, Vol. 20, 1977, p. 2121.

[4] D. A. Hammer, et al., "Collective Beam-Plasma Interaction for REB Passed through Inhomogeneous Low-Density Plasma," IEEE Transactions on Plasma Science, Vol. 7, 1986, p. 83.

[5] P. H. De Hann, et al., "Injection of a Relativistic Electron Beam into Neutral Hydrogen Gas," Physics of Fluids, Vol. 25, 1982, p. 592.

[6] M. Friedman, et al., "High-Power Modulated Intense Relativistic Electron Sources with Applications to RF Generation and Controlled Thermonuclear Fusion," IEEE Transactions on Plasma Science, Vol. 14, 1986, p. 201.

[7] D. Umstadter, "Topical Review: Relativistic Laser-Plasma Interactions," Journal of Physics D: Applied Physics, Vol. 36, 2003, p. 151.

[8] S. L. Voronov, et al., "Control of Laser High-Harmonic Generation with Counter Propagating Light," Physics Review Letter, Vol. 87, 2001, p.133902. doi:10.1103/PhysRevLett.87.133902

[9] D. Dahiya, et al., "Phase-Matched Second- and ThirdHarmonic Generation in Plasmas with Density Ripple," Physics of Plasmas Vol. 14, 2007, p. 123104. doi:10.1063/1.2817088

[10] P. Gibbon, "High Order Harmonic Generation in Plasmas," IEEE Journal of Quantum Electronics, Vol. 33, 1997, p. 1915. doi:10.1109/3.641306

[11] E. C. Jarque and L. Plaja, "Harmonic Filtering in an Optically Laser-Generated Plasma," Physics Review, E, Vol. 58, 1998, p. 7864. doi:10.1103/PhysRevE.58.7864

[12] R. Lichters, et al., "Short- Pulse Laser Harmonics from Oscillating Plasma Surfaces Driven at Relativistic Intensity," Physics of Plasmas, Vol. 3, 1996, p. 3425. doi:10.1063/1.871619

[13] Biedron et al, "The Sensitivity of Nonlinear Harmonic Generation to Electron Beam Quality in Free Electron Lasers," Nuclear Instruments and Methods in Physics Research A, Vol. 483, 2002, p. 1. doi:10.1016/S0168-9002(02)00292-9

[14] P. B. Corkum and F. Krausz, "Attosecond Science," Nature Physics, Vol. 3, 2007, p. 381. doi: $10.1038 /$ nphys 620

[15] G. D. Tsakiris, et al., "Route to Intense Single Attosecond Pulses," New Journal of Physics, Vol. 8, 2006, p. 19. doi:10.1088/1367-2630/8/1/019 
[16] B. Dromey, et al., "High Harmonic Generation in the Relativistic Limit," Nature Physics, Vol. 2, 2006, p. 456. doi:10.1038/nphys338

[17] M E Dieckmann et al., "Simulation Study of the Filamentation of Counter-Streaming Beams of the Electrons and Positrons in Plasmas," Plasma Physics and Controlled Fusion, Vol. 51, 2009, p. 065015.

[18] W. Rozmus et al., "Propagation of High Order Harmonics in Dense Relativistic Plasmas," 32nd EPS Conference on Plasma Phys. Tarragona, 27 June - 1 July 2005 ECA Vol.29C, O-1.006 (2005)

[19] G. I. Pert, "Optimizing the Performance of Nickel-Like Collisionally Pumped X-Ray Lasers. II. Lasers for the Wavelength Range 50--100\AA," Physical Review A, Vol. 75, 2007, p. 023808 .

doi:10.1103/PhysRevA.75.023808

[20] F. Gruener, et al., "Design Considerations for Table-Top, Laser-Based VUV and X-Ray Free Electron Lasers," Applied Physics B, Vol. 86, 2007, p. 431. doi:10.1007/s00340-006-2565-7

[21] Sh. M. Khalil, et al., "Wave Generation in Relativistic Plasma Layer,” Physica Scripta, Vol. 30, 1984, p. 357. doi:10.1088/0031-8949/30/5/012

[22] Sh. M. Khalil and R.N. El-Sherif, "Second Harmonic Generation in Inhomogeneous Anisotropic Plasma Due to Beam- Plasma Interaction," 16th European Conference on Controlled Fusion and Plasma Physics, March, 13-17, Venice, Italy, 13B/IV, 1989, p. 1433.

[23] Sh. M. Khalil and N. G. Zaki, "REB Heating of Inhomogeneous Anisotropic Plasma," International Conference on Phenomena in Ionized Gases, ICPIGXIX, 10-14 July, Belgrade,Yugoslavia, PS/G 4, 1989, p. 906.

[24] J. Krasnoselskikh et al., "Beam-Plasma Interaction in Randomly Inhomogeneous Plasmas" Journal of Geophysical Research, Vol. 112, 2007, p. A10109.

[25] L. N. Vyacheslavov, 30th EPS Conference on Contr. Fusion and Plasma Physics, St. Petersburg, 7-11 July 2003 ECA Vol. 27A, p. 2.24.

[26] V. Krasnoselskikh, and K. Evgen, "Beam Plasma Interaction in Strongly Inhomogeneous Plasma," Geophysical Research Abstracts, Vol. 12, 2010, p. 11744.

[27] Sh. M. Khalil, K. H. El-Shorbagy and E. N. El-Siragy, "Minimizing Energy Losses in Inhomogeneous Plasma Filled Waveguide Using Electron Beam," Contribution to Plasma Physics, Vol. 42, 2002, p. 67. doi:10.1002/1521-3986(200201)42:1<67::AID-CTPP67> 3.0.CO;2-C

[28] Sh. M. Khalil, Y. A. Sayed, K.H. El-Shorbagy and A. T. Gendy, "Field Stability by an Electron Beam in Warm Magnetized Plasma-Filled Waveguide," Proceeding: Bilateral Seminar of the International Bureau, Forschungszentrum - Julich, Germany, Vol. 34, 2004, p. 283. 